\title{
Childhood Cerebellar Astrocytoma
}

National Cancer Institute

\section{Source}

National Cancer Institute. Childhood Cerebellar Astrocytoma. NCI Thesaurus. Code C6286.

Benign and malignant astrocytomas that arise from astrocytes in the cerebellum. More than $80 \%$ of childhood cerebellar astrocytomas are pilocytic astrocytomas which have a favorable prognosis. The remainder are composed of diffuse or fibrillary subtypes with malignant astrocytomas occurring only rarely in the cerebellum during childhood. 